\title{
Improving Health Equity for First Nations, Inuit and Métis People: Ontario's Aboriginal Cancer Strategy II
}

Alethea Kewayosh, Loraine Marrett, Usman Aslam, Richard Steiner, Margaret Moy Lum-Kwong, Joseph Imre and Abigail Amartey

\begin{abstract}
Cancer incidence is increasing more rapidly and cancer survival is worse among Ontario's First Nations, Inuit and Métis (FNIM) populations than among other Ontarians. Cancer Care Ontario's Aboriginal Cancer Strategy II aims to reduce this health inequity and to improve the cancer journey and experience for FNIM people in Ontario. This comprehensive, multi-faceted strategy was developed and is being implemented with and for Aboriginal Peoples in Ontario in a way that honours the Aboriginal Path of Well-being.
\end{abstract}

\section{Introduction}

First Nations, Inuit and Métis (FNIM) people represent the fastest-growing segment of the Canadian population. Between 2006 and 2011, the number of people self-identifying as Aboriginal increased by $20.1 \%$, compared with Canada's overall growth rate of $5.3 \%$; in $2011,1,400,685$ people (representing $4.3 \%$ of the total Canadian population) self-identified as Aboriginal. Ontario has Canada's largest number of Aboriginal people in any province or territory: 301,425 (representing $2.2 \%$ of Ontario's population) or $21.5 \%$ of the country's total Aboriginal population. Within Ontario, 67\% of those reporting an Aboriginal identity are First Nations (FN), 29\% are Métis and just over 4\% are Inuit (Statistics Canada NHS 2011).

FNIM people are the original inhabitants of Canada, possessing rich and diverse traditions, distinct practices and beliefs and unique understandings of health and wellness. FNIM political and governance structures, processes and decision-making practices are also unique to the three nations. Rather than being categorized as an ethnic group, FNIM people are distinct nations constitutionally recognized by the Government of Canada. The Constitution Act (1982, Section 35-1) protects Aboriginal and treaty rights and recognizes that Aboriginal peoples include “... the Indian (First Nations), Inuit and Métis people of Canada” (Aboriginal Affairs and Northern Development Canada 2013a). "First Nation" may refer to specific communities, or as an adjective to describe an individual or a group; the term "First Nation" is often used to replace the terms "bands," or "Indian" (AANDC 2013b). In Ontario, there are 133 First Nation communities (also known as "reserves"), with First Nation governments. Métis people are of mixed First Nation and European ancestry, and their history and culture draws on diverse ancestral origins such as Scottish, Irish, French, Ojibway and Cree (AANDC 2013c). Inuit are Aboriginal people primarily from Northern Canada, such as Nunavut, Northwest Territories, Northern Quebec (Nunavik) and Labrador; there are no Inuit communities in Ontario, but the Inuit that do live in the province predominantly reside in Ottawa (AANDC 2013d).

Health disparities continue to exist between indigenous and non-indigenous populations in developed nations. While there 
has been notable improvement in the United States, Canada and New Zealand, more needs to be done (Cunningham 2011; Ring 2003).

In 2003, the Institute of Medicine released its report called Unequal Treatment: Confronting Racial and Ethnic Disparities in Health Care, noting that "the majority of studies find that racial and ethnic disparities remain even after adjustment for socio-economic differences and other healthcare access related factors" (IOM 2003: 5). The report's authors observed that the evidence pointed to disparities in cancer care and that those disparities were associated with higher death rates among minorities. That same year, in their First Ministers' Accord on Health Renewal, Canada's First Ministers acknowledged the gap in health status between Aboriginal and non-Aboriginal Canadians (Health Canada 2003). Ten years later, the Health Council of Canada reported that "despite significant investments to address inequities in the health status and health outcomes of Aboriginal Peoples, the impact of these initiatives is unclear" (Health Council of Canada May 2013a: 35).

In this paper, we examine the incidence of cancer among FNIM people in Ontario, and we address the social determinants contributing to health inequity in those groups. Next, we outline the Aboriginal Cancer Strategy II (ACS II) (Cancer Care Ontario, 2012), a multi-faceted approach to reducing risk, prevention and improving the cancer journey for Ontario's FNIM people, and we provide details about three of its six priorities. Our paper concludes by discussing several of the policyrelated initiatives that will, in the coming years, help to advance the ACS II's objectives.

\section{LIST OF ACRONYIMS}

\begin{tabular}{ll} 
Acronym & Description \\
\hline FNIM & First Nations, Inuit and Métis \\
FN & First Nations \\
ACS II & Aboriginal Cancer Strategy II \\
MOHLTC & Ministry of Health and Long-Term Care \\
CCO & Cancer Care Ontario \\
JOACC & Joint Ontario Aboriginal Cancer Committee \\
ACCU & Aboriginal Cancer Control Unit \\
PTOs & Political Territorial Organizations \\
SAR & Screening Activity Report \\
RCPS & Regional Cancer Programs \\
ATP & Aboriginal Tobacco Program \\
TFNHSOC & Trilateral First Nations Health Senior Officials \\
& Committee
\end{tabular}

\section{Disparities Affecting Health Equity among FN, Inuit and Métis in Ontario}

\section{Disparities in Cancer Burden}

Globally, cancer mortality is higher for indigenous people. For example, in Australia, cancer mortality for indigenous people is $45 \%$ higher than for other Australians (Whop 2012). In Cottrell's analysis of cancer profiles and survival of Aboriginal and non-Aboriginal people in South Australia, she notes "the survival results reflect the multitude of obstacles confronting Aboriginal patients with cancer compared with non-Aboriginal cancer patients" (Cottrell 2007: 495).

Similarly, in Canada, people with any Aboriginal origins have been found to have higher age-standardized mortality rates than people with no Aboriginal ancestry (Marrett and Chaudhry 2003; Wilkins 2008). Mortality rates have been found to be highest among Registered Indians (those who are registered as Indians under the Indian Act) (Wilkins 2008).

While there are no specific data on the cancer burden among Ontario's Inuit and Métis people, cancer patterns differ significantly between the province's FN people and general populations. Cancer incidence rates for major cancers are increasing more rapidly among FN people and cancer survival is worse in this population compared with other Ontarians (Marrett and Chaudhry 2003; Nishri et al. 2015). For example, while incidence rates for breast cancer have stabilized in the general population, the incidence of breast cancer among Ontario's FN women continues to increase. A recent study examining reasons for poorer breast cancer survival in FN women compared with non-FN Ontarians identified later stage at diagnosis and greater co-morbidity as the two major determinants of survival differences (Nishri et al. 2015; Sheppard et al. 2010), There are also major disparities in cancer risk factors, with, for example, significantly higher rates of cigarette smoking in FN and Métis Ontarians, compared with the non-Aboriginal population (Cancer Care Ontario 2014). These portend a growing cancer burden in Aboriginal Ontarians and growing inequalities.

\section{Disparities in the Social Determinants of Health}

Broad determinants of health contribute to the unique cancer burden faced by Ontario's FNIM people. Generally, FNIM people are younger, more mobile, less educated and more often unemployed than non-Aboriginal people. They also earn less and are more likely to be incarcerated, and there are proportionally more Aboriginal single-parent families compared with non-Aboriginal families ( $34.4 \%$ vs. $17.4 \%$, respectively) (Statistics Canada 2011). The median income per Aboriginal household is $\$ 46,865$, which is significantly lower than Ontario's median household income of $\$ 73,290$. The Aboriginal unemployment rate in Ontario is $12.3 \%$, and about $57 \%$ of the FNIM population over 15 years of age is working. Conversely, 
the provincial rate of unemployment is $7.2 \%$, representing almost half the level of unemployment among FNIM Ontarians (HRSDC 2013). Thirty-eight percent of FNIM Ontarians have no post-secondary certificate, diploma or degree, and $61.8 \%$ have only a high school diploma or less.

Other socio-cultural factors, such as poor housing, language barriers, cultural differences and geographic isolation, likely also contribute to health-related disparities (Health Council of Canada 2013a).Twenty-eight (21\%) of the $133 \mathrm{FN}$ communities in Ontario are isolated (i.e., no regular flights, no year-round road access), with an approximate total population of 90,000 individuals. Thirteen of those 28 remote FN communities are accessible only by air, making it difficult to procure healthy, affordable food and access mainstream health services. With limited healthcare services in many communities, medical travel is a frequent necessity; however, given the higher unemployment and lower income levels, medical travel costs can be prohibitive.

Many FNIM people delay seeing a healthcare professional until they are seriously ill because they are afraid their diagnosis will mean they will be sent away for care and never return. If they do seek help and the care is not culturally safe, they may not return for followup appointments or continue with their treatment plans (Health Council of Canada 2013a). Jurisdictional issues of federal versus provincial accountability also arise in the provision of healthcare for $\mathrm{FN}$ people living on-reserve.

\section{Cancer Care Ontario's Aboriginal Cancer Strategy II}

In Ontario today, the equitable distribution of healthcare is set within the 2010 Excellent Care for All Act, which established the foundation for improving healthcare quality and affirmed the value of a patient-centred health system. In keeping with this statute, Ontario's Ministry of Health and Long-Term Care (MOHLTC 2013) developed a Health Equity Impact Assessment tool to support improved health equity and identified FNIM people as a group that may experience significant unintended health impacts as a result of policies, programs and initiatives.

One of Cancer Care Ontario's (CCO) goals is to improve the performance of the cancer system with, and for Aboriginal people in Ontario in a way that honours the Aboriginal Path of Well-being (Figure 1). The Aboriginal Path of Well-being involves: a balance and harmony between and within the four aspects of a person's nature, which are mental, physical, spiritual and emotional; responsibility for each individual's own health and lifestyle choices; respect for others and the application of traditional Aboriginal practices with integrity, acceptance and empowerment; and an understanding of individual growth and development as it pertains to the Path. Whereas traditionally the basis for the Western health model could be seen as linear and disease- or illness-focused, the Aboriginal model by contrast involves a circular perspective and a focus on health and wellness (Stewart 2014). The steps to the Aboriginal Path of Well-being include, but are not limited to, the following aspects (Hill 2008):

\section{FIGURE 1.}

\section{The Aboriginal Path of Well-being}

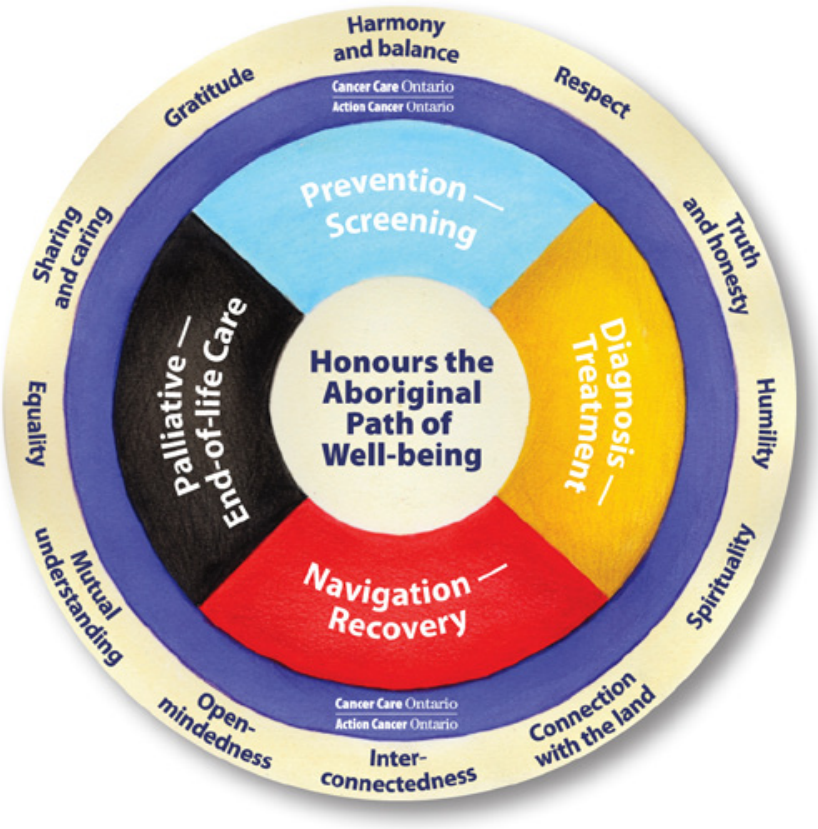

1. Health in balance: giving equal importance to all aspects of health

2. Wellness: both emotional and spiritual

3. Active choice: one's ownership for health decisions

4. Holistic approach: balance the mind, body and spirit with community and environment

5. Understand root causes: past and present aspects that impact health

6. Joint and personal responsibility: health and well-being is the responsibility of the individual, family and community

The data addressed above pertaining to disparities affecting the health of FNIM people underscore the need for a specific FNIM-focused cancer control strategy that includes both improvements to surveillance and research data in order to better document the current state and progress, as well as actions to improve cancer control across the cancer journey. Launched in 2012, the ACS II is a component of the broader Ontario Cancer Plan and builds on the foundation of the first Aboriginal Cancer Strategy (2004-2009). The ACS II aims to adhere to the principles of the Aboriginal Path of Well-being, while establishing an Aboriginal cancer system that is community-based, holistic in its approach, culturally competent and inclusive. 
Acknowledging that FNIM people are distinct, constitutionally recognized groups with Aboriginal and treaty rights, $\mathrm{CCO}$ engaged directly with individual FNIM nations, communities and organizations across Ontario to develop the ACS II. This extensive engagement to establish strong, positive relationships with FNIM groups and communities was designed to ensure a shared agenda that extends beyond the strategy's development.

Building on this collaborative network, in the two years since its launch, the ACS II has been used to formalize the communication and engagement structures necessary to maintain and achieve strategic objectives within a framework of mutual respect, recognition of traditions and practices and honour for the history, culture and diversity of FNIM people. A Joint Ontario Aboriginal Cancer Committee (JOACC) is in place, with representatives from each of the FNIM nations and organizations. Its mandate is to ensure engagement and partnerships with FNIM groups and communities, build trust and a shared agenda, improve accountability and ensure an FNIM-centric perspective in the identification, development and implementation of solutions. The ACS II is supported by the wisdom and guidance of FNIM leaders, elders, cancer survivors and the knowledge and expertise of FNIM health program leads and other health professionals. The core strength of the ACS II is that it is not a pan-Aboriginal initiative, but rather an engagement tool that facilitates direct engagement with FNIM leaders and health networks, recognizing and respecting the differences between diverse and distinct Aboriginal cultures.

The ACS II comprises the following six strategic priorities:

1. Building productive relationships

2. Research and surveillance

3. Prevention

4. Cancer screening

5. Palliative care and supportive care

6. Education

In this paper we focus on three of the six strategic priorities: building productive relationships, research and surveillance and prevention.

\section{Building Productive Relationships}

Building productive relationships is the ACS II's first strategic priority. These relationships based on respect and understanding are critical to the success of the ACS II. The foundation is the Relationship Protocols between CCO and each of the FNIM organizations and governance structures, including Political Territorial Organizations (PTOs). Relationship Protocols are agreements that acknowledge the unique history and ways of life of FNIM communities in Ontario, and establish and formalize the collaborative relationship between CCO and FNIM people.
These protocols build trust and mutual respect, and create mutual accountability.

In 2013, two Relationship Protocols with CCO were signed: Grand Council Treaty \#3 (May 2013) and the Union of Ontario Indians (June 2013). In 2014, two further Relationship Protocols with CCO were signed: The Ontario Federation of Indigenous Friendship Centres (July 2014) and the Nishnawbe Aski Nation (August 2014). The approval and signing of the remaining Relationship Protocols are anticipated in 2015, including the Association of Iroquois and Allied Indians, the Métis Nation of Ontario and with Inuit health service providers in the Ottawa region.

Understanding and adhering to FNIM protocol and governance structures is a vital component of working respectfully, and effectively, with FNIM communities. When considering specific initiatives with FN, CCO will seek the guidance and direction of the First Nation Leadership, through the PTO and, when community-specific, through the Chief and Council. This ensures that $\mathrm{CCO}$ has the full, prior and informed approval of the political leadership in each community before any work begins.

This approach was important in the development of a community-based colorectal cancer Screening Activity Report, which helps to ensure that residents are up-to-date with their colorectal cancer screening, and can facilitate early identification and prevention of colorectal cancer. CCO provided an overview of the project and sought community approval and support throughout the project. Engagement of the community's Chief and Council ensured greater awareness and recognition to address colorectal cancer screening in the community. Key resources and community members were engaged and aware of the project and importance of cancer screening. The success of this project was demonstrated in positive feedback received from community leadership, physicians and healthcare team members who found the approach of community engagement as respectful and effective. The report was noted as an important tool to support cancer screening in the community.

Establishing the roles of Aboriginal Cancer Leads and Aboriginal Navigators in 10 of Ontario's 13 Regional Cancer Programs (RCPs) with a higher FNIM population also helps to ensure local implementation and capacity to establish local solutions to address FNIM barriers to care. The Aboriginal Cancer Lead is the local champion in the implementation of the ACS II in each health region. The development of Regional Aboriginal Cancer Plans, through close partnerships between CCO, the RCPs and FNIM health tables (e.g., boards, advisory committees and community groups), ensures that each Regional Aboriginal Cancer Plan addresses the unique challenges and barriers faced by FNIM people in accessing cancer-related services. This collaborative approach is designed not only to make the cancer system function more effectively for FNIM 
people in each region, but also to ensure ongoing respectful, sustainable and productive relationships and an FNIM voice in the planning of the delivery of cancer services in each region.

The role of the Aboriginal Navigator is founded on the recommendations following CCO's Aboriginal Cancer Care Needs Assessment (2002), to provide support and advocacy for Aboriginal patients and their family members, to address and promote awareness of the cultural needs of Aboriginal clients and to network with Aboriginal and non-Aboriginal health groups and organizations. The Health Council of Canada (2012) has identified growing evidence demonstrating that the use of navigators can improve access to Western and traditional health services; increase quality of care; increase knowledge, understanding and adherence to recommended treatments; improve trust of providers; enhance coordination of care; and increase patient satisfaction. Similar evidence has been demonstrated in other jurisdictions. In British Columbia, Aboriginal Patient Navigators have been credited with an increasing access to care through increasing community referrals to hospitals from $24 \%$ to $40 \%$ (Health Canada 2011). The establishment of Aboriginal Patient Navigators in Quebec was noted to "improve awareness of Inuit health issues among provincial staff, and improved trust and communication between service providers" (Health Canada 2011). Aboriginal Patient Navigators in Newfoundland have demonstrated reduction in patient stress/anxiety and enhanced coordination after care (Health Council of Canada 2012).

\section{Research and Surveillance}

The ACS II recognizes that data gathered through research and surveillance are needed to support the identification of priorities for action, strategy development and resource planning. Recent work using population-based survey data has been able to provide a clearer picture of the current cancer risk factor and screening participation profiles among Ontario's FN and Métis groups separately, as well as in comparison with the province's non-Aboriginal population. Currently, however, limited surveillance data are available on cancer in Ontario's FNIM communities, and the information that does exist is generally not up-to-date. There is also a paucity of research data about why inequalities exist and what interventions are effective to reduce them.

\section{Prevention}

Prevention offers the most cost-effective, long-term cancer control strategy. At least one-third of all cancer cases are preventable, and tobacco use is the single greatest avoidable risk factor for cancer mortality (WHO 2013).

Tobacco in Aboriginal communities has moved from its traditional ceremonial use to non-traditional, non-ceremonial use. Sacred tobacco represents a vital component of traditional practice for FN and Métis, dating back thousands of years. It is free of chemicals, held in the hand and offered to the earth and fire; it is not smoked. In many FN cultures, tobacco is a symbol of peace that is respected and honoured. It is one of the four sacred medicines (the other three are sage, cedar and sweetgrass)

FIGURE 2.

Percentage of population who are current cigarette smokers - Ontario adults (aged 20+), by Aboriginal identity (off-reserve) and by sex, 2007-2011 combined

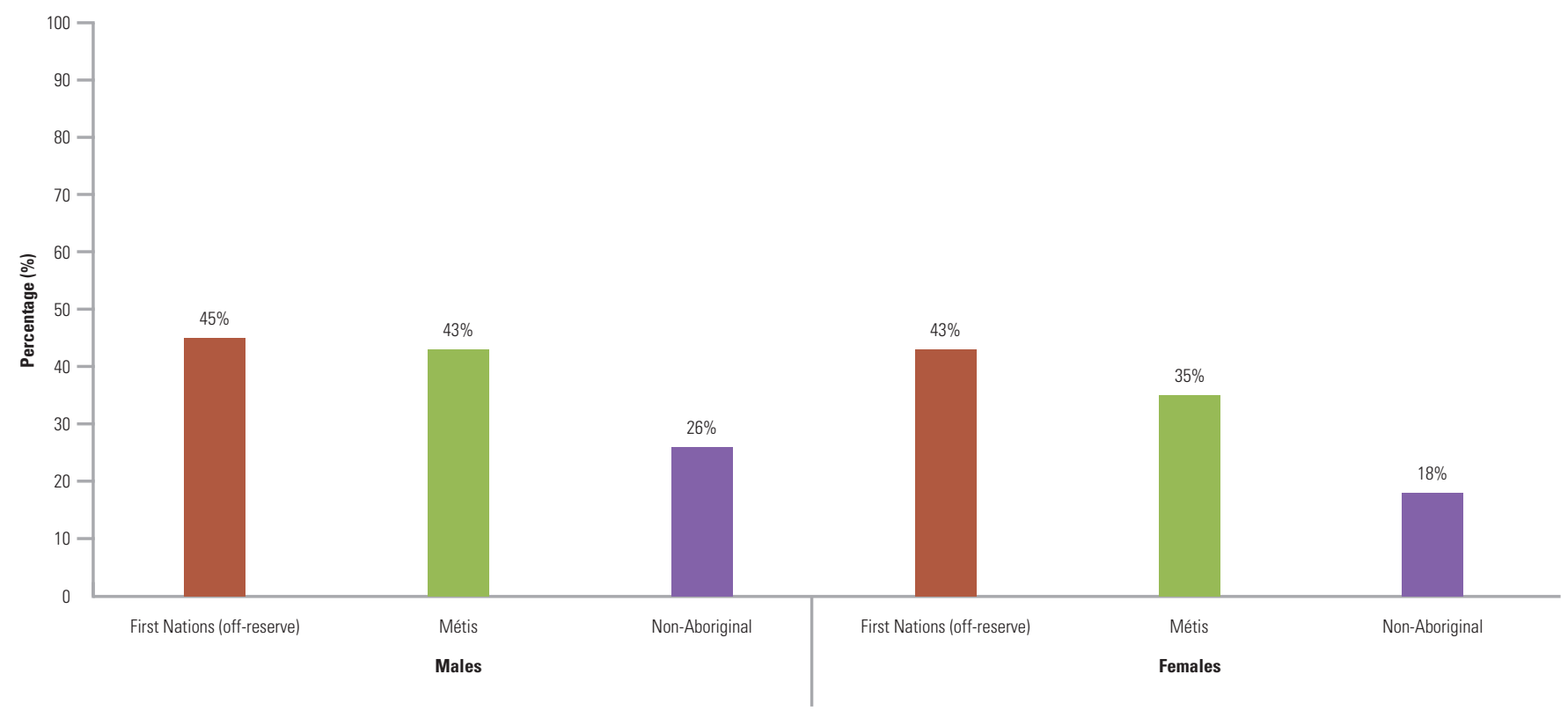


that are understood by FN people to have been offered to them by the Creator to assist them in their journey of life.

In Ontario, less than $18 \%$ of female and $26 \%$ of males report they are current smokers. However, the rate of non-traditional tobacco use among FNIM communities is as high as $49 \%$, and Canada's Inuit have the highest lung cancer rates in the world (Physicians for a Smoke-Free Canada 2013). The rate of cigarette smoking is significantly greater among off-reserve FN and Métis people than among non-Aboriginal Ontarians (Figure 2). Furthermore, second-hand smoke exposure reported in the home, car or in public is significantly more common among off-reserve FN males and Métis females than non-Aboriginal Ontarians (Figure 3).

Combating the high prevalence of non-traditional tobacco use and exposure in FNIM communities requires culturally appropriate awareness and education initiatives. The primary goal of CCO's Aboriginal Tobacco Program is, therefore, to build capacity towards Tobacco-Wise FNIM communities, among both FNIM and non-FNIM policymakers, healthcare administrators and healthcare and social-care practitioners. A Tobacco-Wise community is one that understands the difference between commercial and sacred tobacco, has initiated discussions around smoke-free policies in the community and, once Tobacco-Wise, implements smoke-free policies. TobaccoWise leads advise and assist communities in designing their own non-traditional tobacco awareness and cessation programs. They also forge links with various local/community initiatives, such as the Tobacco-Wise Little Native Hockey League youth campaign to raise awareness among young people about the misuse of tobacco. In addition, smoking-cessation messages are

\section{FIGURE 3.}

Proportion of population who are exposed to second-hand smoke (in home, car or public) - Ontario adults (aged 20+), by Aboriginal identity (off-reserve) and by sex, 2007-2011 combined

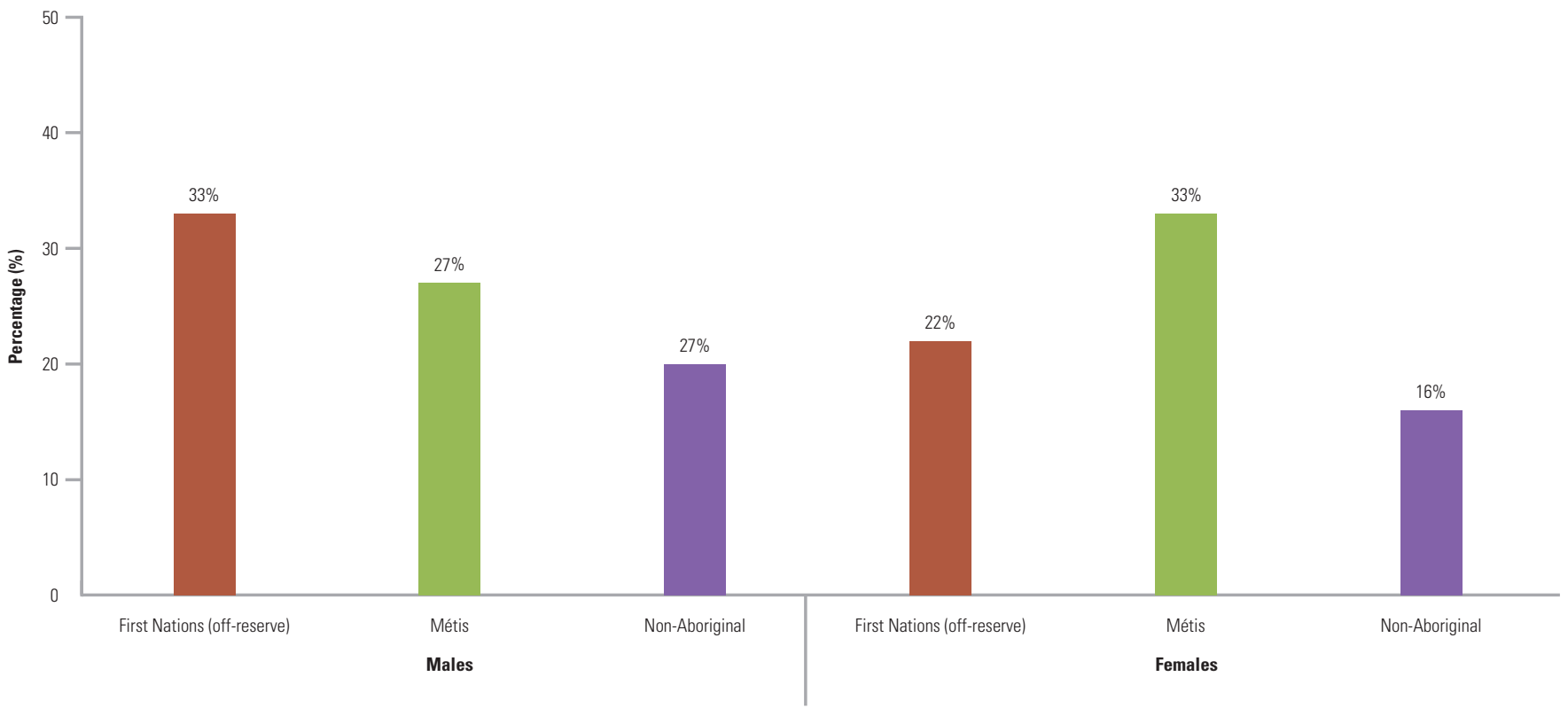

broadcast (in translation) on local radio stations, which is an important conduit to remote communities.

\section{The Way Forward: Opportunities and Challenges}

Improving the cancer journey for people in Ontario cannot be achieved overnight. It is a complex issue with many interconnected parts, and a multi-pronged approach that embeds an FNIM perspective is required to address this complex system issue.

The Aboriginal Path of Well-being outlined above incorporates a holistic understanding of the individual that is tied to FNIM values and traditions, and can be used as a framework for patient- and community-centred care.

A broader discussion regarding program and policy changes involving all levels of government is needed to ensure that cancer care is more equitable and accessible - from prevention and First Nations Health Senior Officials Committee (TFNHSOC) was established in Ontario, aimed at enabling the three levels of government (Chiefs of Ontario and the provincial and federal governments) to collaborate on shared priorities. To date, these priorities include mental health and addictions, public health, data management and chronic disease prevention (with a focus on diabetes and cancer).

Further, there is an opportunity to develop regional capacity and more responsive programs and services by taking a more collaborative partnership approach with FNIM people than in the past. FNIM patients and families would function as design and quality improvement partners in the co-creation of screening to treatment and palliative care. In 2011, the Trilateral 
programs and services. Balik (2012: 12) notes that this "doing with" model requires leadership, partnerships and infrastructure, "essential factors in the transformation from an organizational-centred focus to a patient-and-family-centred one."

Finally, improving the cancer journey for FNIM people requires improved surveillance and research. Extended surveillance data will allow for the identification of additional important research questions and, ultimately, promote actions to improve FNIM cancer control. To enable this, a number of data barriers will need to be addressed, including FNIM identification and community privacy. A key priority is, therefore, to identify FNIM data sources and assess their viability for integration into the current Ontario cancer data holdings for analysis.

At the same time, FNIM governance and self-government have implications for jurisdiction and control over FNIM-related research and information. According to the First Nations Information Governance Centre, "The First Nations Principles of OCAPTM (ownership, control, access, and possession) means that First Nations control data collection processes in their communities. First Nations own, protect and control how their information is used," (www.fnigc.cal ocap.html). A solution will need, therefore, to be mutually established between CCO and FNIM communities, organizations, data owners and data stewards of the various data holdings in order to develop an FNIM-identified surveillance system that permits FNIM-specific cancer research and control initiatives to be implemented. Doing so will require acknowledgement of the FNIM approach to privacy, which, unlike the standard Western industrialized model, emphasizes "community privacy" and group interests, while collaboratively addressing privacy concerns. HQ

\section{About the Authors \\ Alethea Kewayosh is the director of the Aboriginal Cancer Control Unit at Cancer Care Ontario (CCO). She led the development and launch of the Aboriginal Cancer Strategy II (ACS II).}

Loraine Marrett, PhD, is a senior scientist with CCO's Aboriginal Cancer Control Unit. Her work includes surveillance of cancer and cancer risk factors, as well as the use of data to drive system change.

Usman Aslam, MPH, is a senior manager with CCO's Aboriginal Cancer Control Unit. He has accountability for all ACS II projects and initiatives.

Richard Steiner, MA, is a partnership liaison officer with CCO's Aboriginal Cancer Control Unit. He acts as a liaison between FNIM health networks and the Regional Cancer Programs and helps to fulfill CCO's commitment to building productive relationships with FNIM people.

Margaret Moy Lum-Kwong, BScN, MBA, CHE, is a former senior manager with CCO's Aboriginal Cancer Control Unit. She had accountability for selected ACS II projects and initiatives.
Joseph Imre, MA, is a senior project analyst with CCO's Aboriginal Cancer Control Unit. He supports the implementation of the ACS II through strengthening relationships with First Nations, Inuit and Métis populations.

Abigail Amartey, BSc, MPH, is a research associate with CCO's Aboriginal Cancer Control Unit. She focuses on analyzing cancer incidence, mortality, risk factors and screening uptake.

\section{References}

Aboriginal Affairs and Northern Development Canada (AANDC). (2013a). Retrieved October 3, 2014. <https://www.aadnc-aandc.gc.ca/ eng/1100100013785/1304467449155>.

Aboriginal Affairs and Northern Development Canada (AANDC). (2013b). "First Nations". Retrieved October 3, 2014. <https://www. aadnc-aandc.gc.ca/eng/1100100013791/1100100013795>.

Aboriginal Affairs and Northern Development Canada (AANDC). (2013c). "Métis". Retrieved October 3, 2014. <https://www.aadncaandc.gc.ca/eng/1100100014427/1346434788986>

Aboriginal Affairs and Northern Development Canada (AANDC). (2013d). "Inuit". Retrieved October 3, 2014. <https://www.aadncaandc.gc.ca/eng/1100100014187/1100100014191>.

Balik, B. 2012. "Patient-and Family-Centredness: Growing a Sustainable Culture.” Healthcare Quarterly 15(Special Issue): 10-12. doi:10.12927/hcq.2012.23154

Cancer Care Ontario. 2012. "Aboriginal Cancer Strategy II (ACS II).” Retrieved December 1, 2014. <https://www.cancercare.on.ca/ common/pages/UserFile.aspx?fileId=138007>.

Cancer Care Ontario. 2014. "Aboriginal Cancer Strategy II Annual Report 2012-2013: Staying On the Path." Retrieved December 1, 2014. <https://www.cancercare.on.ca/common/pages/UserFile. aspx? fileId $=297708>$.

Cottrell, J., J. Street, A. Chong and D. Roder. 2007. "Comparing Cancer Profiles and Survival of Aboriginal and Non-Aboriginal Patients in South Australia: Where are the Opportunities for Improving Aboriginal Health?" Asian Pacific Journal of Cancer Prevention 8: 495-501.

Cunningham, M. 2011. State of the World's Indigenous Peoples. United Nations, Department of Economic and Social Affairs, Chapter 5. Retrieved January 17, 2014. <www.un.org/esa/socdev/unpfii/ documents/SOWIP_chapter5.pdf $>$.

Health Canada. 2003. First Ministers' Accord on Health Care Renewal. Retrieved online December 1, 2014. <http://www.hc-sc.gc.ca/hcs-sss/ delivery-prestation/fptcollab/2003accord/index-eng.php>.

Health Canada. 2011. "ARCHIVED - Aboriginal Health Transition Fund: Outcomes and Lessons Learned." Retrieved December 4, 2013. <http:/www.hc-sc.gc.ca/fniah-spnia/services/acces/oll-rlr-eng.php>.

Health Council of Canada. 2012. "Empathy, Dignity, and Respect. Creating Cultural Safety for Aboriginal People in Urban Health Care." Retrieved December 4, 2013. <http://www.abo-peoples.org/ wp-content/uploads/2012/10/Aboriginal_Report_EN_web_final. pdf>.

Health Council of Canada. 2013a. "Progress Report 2013: Health Care Renewal in Canada." Retrieved December 3, 2013. <http://www. healthcouncilcanada.ca/rpt_det.php?id=481>

Health Council of Canada. 2013a. "Canada's Most Vulnerable: Improving Health Care for First Nations, Inuit and Métis Seniors.” 
Retrieved December 4, 2013. <http://www.healthcouncilcanada.ca/ content_ab.php?mnu $=2 \& \mathrm{mnu} 1=48 \& \mathrm{mnu} 2=30 \& \mathrm{mnu} 3=55>$. Health Council of Canada. 2013b. Retrieved December 15, 2013. http:// www.healthcouncilcanada.ca/accord.php?mnu=1\&mnu1=7.

Hill, L.P. 2008. "Understanding Indigenous Canadian Traditional Health and Healing". Retrieved October 3, 2014. <http://scholars. wlu.ca/>.

Human Resources and Skills Development Canada (HRSDC). 2013. "Aboriginal Labour Market Bulletin.” Retrieved December 2013. <http://www.hrsdc.gc.ca/eng/jobs/aboriginal/almb.pdf>.

Institute of Medicine (IOM). 2003. In B.D. Smedley, A.Y. Stith and A.R. Nelson, eds., Unequal Treatment: Confronting Racial and Ethnic Disparities in Health Care. National Academies Press. Retrieved December 2013. <http://www.nap.edu/catalog/10260.html>.

Marrett, L.D. and M. Chaudhry. 2003. "Cancer Incidence and Mortality in Ontario First Nations, 1968-1991 (Canada)." Cancer Causes Control 14(3): 259-68.

Ministry of Health and Long-Term Care (MOHLTC). 2013. "Health Equity Impact Assessment Template.” Retrieved November 27, 2013. $<$ http://www.health.gov.on.ca/en/pro/programs/heia/tool.aspx>.

Nishri, E.D., A.J. Sheppard, D.R. Withrow and L.D. Marrett. 2015. "Cancer Survival among First Nations People of Ontario, Canada (1968-2007)." International Journal of Cancer 136(3):639-45. doi: 10.1002/ijc.29024 [Epub ahead of print]

Physicians for a Smoke-Free Canada. 2013. "Smoking among Aboriginal Canadians.” <http://www.smoke-free.ca/factsheets/pdf/ cchs/aboriginal.pdf>.

Ring, I. 2003. "The Health Status of Indigenous Peoples and Others." BMJ 327(7412): 404-05. doi: 10.1136/bmj.327.7412.404.
Sheppard, A.J., A.M. Chiarelli, L.D. Marrett, L. Mirea, E.D. Nishri, M.E. Trudeau, ME., Aboriginal Breast Cancer Study Group. 2010. "Detection of Later Stage Breast Cancer in First Nations women in Ontario, Canada." Canadian Journal of Public Health Jan-Feb; 101(1):101-5.

Statistics Canada. 2011a. 2011 Census. Retrieved July 2013. <http:// www12.statcan.gc.ca/census-recensement/index-eng.cfm $>$. Statistics Canada. 2011b. "Aboriginals in Canada: First Nations People, Métis and Inuit." Retrieved July 2013. <http://www12.statcan.gc.ca/ nhs-enm/2011/as-sa/99-011-x/99-011-x2011001-eng.cfm>.

Stewart, S.L. 2014. "Intergenerational Trauma: Indigenous Perspectives on Healing and Health Care”. Retrieved October 4, 2014 <http:// www.traumatalks.ca/2014>

Whop, L.J., P.C. Valery, V.L. Beesley, S.P. Moore, K. Lokuge, C. Jacka et al. 2012. "Navigating the Cancer Journey: A Review of Patient Navigator Programs for Indigenous Cancer Patients." Asia-Pacific Journal of Clinical Oncology 8: e89-96. doi:10.1111/j.17437563.2012.01532.x.

World Health Organization (WHO). 2013. Cancer Prevention. Retrieved October 31, 2013. <http://www.who.int/cancer/prevention/en/s.

Wilkins, R., M. Tjepkema, C. Mustard and R. Choinière. 2008. "The Canadian Census Mortality Follow-up Study, 1991 through 2001." Statistics Canada, Catalogue no. 82-003-XPE. Health Reports 19(3). Retrieved January 10, 2014. <http://nclc203seminarf.pbworks.com/w/ file/fetch/36460282/Rotterman-2008.pdf\#page $=27>$. 